\title{
Effect of mild hypothermia on renal ischemia/reperfusion injury after cardiopulmonary resuscitation in a swine model $^{1}$
}

\author{
Wei Yuan', Jun-Yuan Wu", Yong-Zhen Zhao'"', Jie Li"', Jie-Bin Li"', Zhen-Hua Li'", Chun-Sheng Li"
}

'Master, Department of Emergency, Beijing Chao-Yang Hospital, Capital Medical University, Beijing, China. Conception and design of the study, acquisition and interpretation of data, manuscript writing.

"PhD, Department of Emergency, Beijing Chao-Yang Hospital, Capital Medical University, Beijing, China. Acquisition of data, critical revision.

I'Master, Department of Emergency, Beijing Chao-Yang Hospital, Capital Medical University, Beijing, China. Acquisition of data.

IVFull Professor, Beijing Key Laboratory of Cardiopulmonary Cerebral Resuscitation, China. Design and supervised all phases of the study, critical revision.

\begin{abstract}
Purpose: To investigate the effect of intravascular cooling on renal function after resuscitation.

Methods: Twenty four pigs were randomized into three groups ( $n=8$ in each group): therapeutic hypothermia group (TH group), normothermia group ( $\mathrm{NH}$ group) and sham operation group (SHAM group). After 6 minutes of untreated VF, CPR was performed. Upon ROSC, the TH group received the intravascular cooling. The NH and SHAM group did not undergo therapeutic hypothermia. Haemodynamic parameters were recorded. The bloods were analyzed for serum creatinine ( $\mathrm{s} C r$ ), CysC and NGAL. The kidney was surgically removed observe pathologic changes under a light microscope.

Results: The sCr increased in both TH and NH groups after ROSC, compared to baseline. Between two groups, the $\mathrm{s} C \mathrm{r}$ and creatinine clearance (Cc) showed lower level in the $\mathrm{TH}$ group. The urine volume per hour in the TH group were higher during cooling. After resuscitation, NGAL and CysC in the NH group were higher than in the TH group. Under the light microscope, compared with the $\mathrm{TH}$ group, the renal injury was prominent in the $\mathrm{NH}$ group. Conclusion: Mild hypothermia had a protection to renal ischemia reperfusion injury after resuscitation.

Key words: Hypothermia. Ischemia. Reperfusion. Kidney. Cardiopulmonary Resuscitation. Swine.
\end{abstract}




\section{Introduction}

After resuscitated cardiac arrest, myocardial dysfunction and the systemic ischemia/reperfusion response can lead to postcardiac arrest syndrome (PCAS). The latter is characterized by the activation of immunologic and coagulation pathways and the release of inflammatory mediators, all of which lead to tissue hypoperfusion and multiple-organ dysfunction ${ }^{1}$. Acute kidney injury (AKI) is a feature of PCAS commonly observed in patients resuscitated from cardiac arrest ${ }^{2,3}$. Therapeutic hypothermia $(\mathrm{TH})$ can reduce a patient's core temperature in an attempt to protect an organ at risk of injury ${ }^{4-6}$. Some previous studies suggest a potential benefit of hypothermia for kidney injury, but both animal and clinical evidence is inconclusive ${ }^{7-9}$. Moreover, even though the RIFLE criteria (risk, injury, failure, loss, end stage) is extensively used in diagnosis and classification of AKI. However, as the systemic state changes under hypothermia, both serum creatinine $(\mathrm{sCr})$ and urine output have a lack of sensitivity and/or specificity. AKI biomarkers may be a better choice, because they can facilitate early diagnosis, provide valuable insight into risk stratification for the vulnerable patients, and also could be used to guide clinical intervention ${ }^{10}$. In the present study, we therefore attempted to utilize neutrophil gelatinase-associated lipocalin (NGAL) and cystatin C (CysC) as novel biomarkers of acute kidney injury to evaluate the effect of intravascular cooling on renal function after resuscitation.

\section{Methods}

The study experimental protocol was approved by the Committee on the Ethics of Animal Experiments of Capital Medical University (Permit Number: 2010-D-013). The animal experiments were in compliance with the Guiding Principles for the Care and Use of Animals expressed in the Declaration of Helsinki ${ }^{11}$.

Twenty-four male Beijing Landrace pigs were used for this experiment. The pigs were $12 \pm 2$ months of age, weighed $31 \pm 2.4 \mathrm{~kg}$ and were provided by a registered laboratory animal center in Beijing, China. The animals were preoperatively fasted overnight with free access to water before surgery.

\section{Anesthesia and perioperative management}

Twenty four pigs were randomized into three groups $(n=8)$ : therapeutic hypothermia group ( $\mathrm{TH}$ group), normothermia group (NH group) and sham

operation group (SHAM group).The pigs were secured in the supine position on the operation table after receiving $2 \mathrm{mg} / \mathrm{kg}$ propofol anesthesia via an ear vein injection. An initial dose of $30 \mathrm{mg} / \mathrm{kg} / \mathrm{h}$ pentobarbital sodium was administered, followed by continuous intravenous maintenance anesthesia using $8 \mathrm{mg} / \mathrm{kg} / \mathrm{h}$ pentobarbital sodium. The pigs received a tracheotomy, tracheal intubation, and ventilation support (Drager-Evata IV; Dreger, Germany) with room air, a ventilation frequency of 12 to 20 breaths/ minute and a fraction of inspiration $\mathrm{O}_{2}\left(\mathrm{FiO}_{2}\right)$ of 0.21 . The tidal volume and ventilation rate were adjusted to maintain normocapnia (endtidal carbon dioxide partial pressure $\left(\mathrm{PCO}_{2}\right)$ of 35 to $45 \mathrm{mmHg}$ ), which was monitored with an infrared capnometer (Model NPB-75, Nellcor Puritan Bennett Inc, Pleasanton, CA).

\section{Electrocardiography (ECG)}

All hemodynamic parameters were monitored with a patient monitoring system (M1165; Hewlett-Packard, Palo Alto, CA, USA). The arterial and central venous catheters were connected to an integrated bedside monitor (PiCCO; Pulsion Medical Systems, Munich, 
Germany) for continuous hemodynamic monitoring including cardiac output (CO) and mean arterial blood pressure (MAP). A catheter was retrogradely inserted into the right internal jugular vein for venous blood collection. The cooling catheter was inserted for intravascular cooling via the right femoral vein, and a vesical catheter with a thermometric detector was intubated into the bladder after fistulation to measure the core body temperature. The other end of vesical catheter is connected with the urine collection bags to monitor urine output simultaneously. All operations were performed using aseptic surgical techniques. The room temperature was maintained at $25^{\circ} \mathrm{C}$ to $26^{\circ} \mathrm{C}$.

\section{Experimental protocol}

Sinus rhythm was measured by ECG after ensuring that the pigs were stable. In the $\mathrm{TH}$ and $\mathrm{NH}$ groups, a medical programmable stimulator (GY-600A, Kaifeng South China Instrument Co., HeNan, China) and oesophageal stethoscope output (S1/S2) (300/200 ms) were used to trigger VF with continuous electrical stimulation consisting of 10 -ms steps $(8: 1)^{12}$. The diagnostic criteria for VF included a rapid decrease in arterial blood pressure and ECG waveforms characteristic of VF. Once VF occurred, mechanical ventilation was discontinued. After 6 minutes of untreated $\mathrm{VF}$, a compression-to-ventilation ratio of $30: 2$ CPR with manual external chest compressions was performed. The quality of CPR, rates of compressions, and defibrillation shocks were maintained using a HeartStart MRx Monitor/ Defibrillator (M3535A; Philips Medical Systems, Best, Holland), which helped maintain chest compression rates at 100 120 beats per minute and the compression depth at 50 60 mm with complete release $^{13}$. During CPR, $100 \%$ oxygen was delivered with the same baseline ventilator settings, and no epinephrine was administered. After 2 minutes of CPR, a single 150J biphasic electrical shock was attempted with a Smart
Biphasic defibrillator (Philips Medical Systems, Andover, MA, USA). If VF persisted, then CPR was resumed for 2 minutes, and $200 \mathrm{~J}$ was used for the second and all subsequent defibrillation attempts. Resuscitation procedures were terminated if pigs had no ROSC after 15 minutes of CPR. ROSC was defined as 10 consecutive minutes of maintenance of systolic blood pressure at $50 \mathrm{mmHg}$. If spontaneous circulation was not restored after 15 minutes of $C P R$, the pig was considered dead ${ }^{14}$. After ROSC, mechanical ventilation was resumed using the same settings as before induction of VF. The sham group only collected blood and urine samples without inducing VF. The samples and haemodynamic parameters were collected at 10 mins before VF induced as the baseline values. From 4 hours before VF induced, the urine was collected to calculateurine output in three groups. All groups received a infusion of intravenous normal saline $(30 \mathrm{ml} / \mathrm{kg})$ at a constant speed from 4 hours before VF to ROSC $24 h$.

\section{Mild hypothermia procedure}

Upon ROSC, the TH group received the intravascular cooling (target temperature $33^{\circ} \mathrm{C} /$ maintained for 12 hours) and then were passively restored to $37^{\circ} \mathrm{C}\left(0.5^{\circ} \mathrm{C} /\right.$ hour $)$ using an intravascular cooling instrument (Thermogard XP; ZOLL Medical Corporation, Los Angeles, CA). It controls the temperature of the saline circulating through the catheter balloons via remote sensing of the animal's temperature. The animal is cooled or warmed as venous blood passes over each balloon - exchanging heat without infusing saline into the animal. The NH and SHAM group did not undergo therapeutic hypothermia. During the induction and maintenance of mild hypothermia, the pigs received pancuronium bromide $(0.1 \mathrm{mg} / \mathrm{kg}$, intravenously) to prevent shivering and muscle movement. Administration was repeated if needed. After the cooling procedure was 
complete, intravascular catheters were removed, incisions were sutured, and the pigs were returned to their cages.

\section{Haemodynamic parameters}

Haemodynamic parameters, including the heart rate $(H R), M A P$, and $C O$ were measured at baseline and at 6, 12 and 24 hours after ROSC.

\section{Blood samples}

Blood samples in the arterialand venous blood were collected at baseline and 6, 12 and 24 hours after ROSC. The blood samples were used to measure serum creatinine $(\mathrm{sCr}), \mathrm{Cys} C$ and NGAL. Blood gases in arterial samples were analysed to determine lactate concentrations.

\section{Urine samples}

The urine was collected separately in 4 time intervals from 4 hours before VF induced, and urine volume per hour was calculated. The urine volume per hour at baseline was calculated from 4 hours before VF to VF. The 4 time intervals were from 4 hours before VF induced to VF, upon ROSC to ROSC $6 \mathrm{~h}$, ROSC $6 \mathrm{~h}$ to $12 \mathrm{~h}$, and ROSC $12 \mathrm{~h}$ to $24 \mathrm{~h}$. The urine samples were used to measure urine creatinine to calculate creatinine clearance $(\mathrm{Cc}$, urine creatinine $x$ urine volume/serum creatinine $x$ time in minutes).

\section{Renal tissue sampling}

The animals were euthanized with propofol (3 $\mathrm{mg} / \mathrm{kg}$, intravenously) followed by $10 \mathrm{~mL}$ of potassium chloride $(10 \mathrm{~mol} / \mathrm{L}$, intravenously) 24 hours after ROSC. The kidney was surgically removed and preserved in $10 \%$ formaldehyde or $4 \%$ paraform aldehyde to observe pathologic changes under a light microscope. A grade of renal injury of semiquantitative evaluation was done in each animal (Table 1$)^{15}$.The pathologic evaluations were performed by an independent pathologist who has more than 10 years of experience and was blinded to this study.

Table 1 - Pathologic change for a semiquantitative evaluation of kidney injury.

\begin{tabular}{|c|c|}
\hline Grade & $\begin{array}{l}\text { Pathologic changes under light } \\
\text { microscope }\end{array}$ \\
\hline 0 - Normal & Renal histopathology \\
\hline 1 - Mild & $\begin{array}{l}\text { Mild interstitial edema, renal } \\
\text { tubular necrosis, slight glomerular } \\
\text { capillaries angiectasis, and } \\
\text { inflammatory cells infiltration }\end{array}$ \\
\hline 2 - Moderate & $\begin{array}{l}\text { Pathologic changes of renal injury } \\
\text { between mild and severe }\end{array}$ \\
\hline 3 - Severe & $\begin{array}{l}\text { Severe interstitial edema, } \\
\text { renal tubular necrosis, severe } \\
\text { glomerular capillaries angiectasis, } \\
\text { mesangial proliferation, and severe } \\
\text { inflammatory cells infiltration }\end{array}$ \\
\hline
\end{tabular}

\section{Statistical analysis}

All analyses were conducted using SPSS 19.0 software (SPSS, Chicago, IL, USA). Data for continuous variables are expressed as the mean \pm s.d. Student's t-test was used for comparisons between every two groups. Differences at different time points were assessed by repeated-measures analysis of variance and the Bonferroni correction for post hoc comparisons between multiple experimental groups. In addition, the continuous variables were fixed to normal distributions and equal variances were analyzed by using the Kolmogorov-Smirnov test and homogeneity of variance test. $\mathrm{P}<0.05$ was defined as statistically significant.

\section{Results}

\section{Core temperature}

From ROSC $1 \mathrm{~h}$ to $24 \mathrm{~h}$, the core temperature increased rapidly in the $\mathrm{NH}$ group 
and fluctuated between $38.6^{\circ} \mathrm{C}$ and $40.2^{\circ} \mathrm{C}$. The core temperature in the $\mathrm{TH}$ group deceased after ROSC, and was lower than that in the $\mathrm{NH}$ group $(P<0.001)$, and reached the target temperature $\left(33.0^{\circ} \mathrm{C}\right)$ at $192 \pm 15 \mathrm{~min}$, and then rewarmed after $12 \mathrm{~h}$. There was no change in the core temperature in the SHAM group (Figure 1).

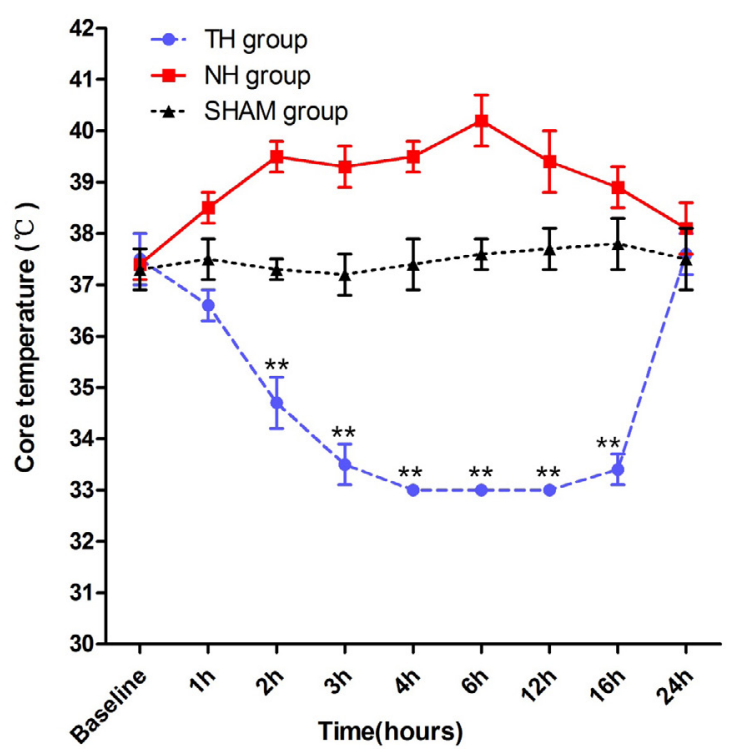

Figure 1 - The core temperature. ROSC: restoration of spontaneous circulation; $\mathrm{TH}$ : therapeutic hypothermia group, $\mathrm{NH}$ : normothermia group; SHAM group: sham operation group. $\mathrm{TH}$ vs. $\mathrm{NH}$ : $* * P<0.001$.

\section{Survival and neurological outcomes}

No significant differences were found for the number of shocks or duration of CPR before ROSC between TH and NH groups. Also, all pigs survived to ROSC $24 \mathrm{~h}$ in these two groups (Table 2).
Table 2 - Comparison of hemodynamic parameters and grade of renal injury between three groups.

\begin{tabular}{lllll}
\hline Outcomes & TH(n=8) & $\begin{array}{l}\text { NH } \\
(n=8)\end{array}$ & t & P \\
\hline $\begin{array}{l}\text { Shocks } \\
\text { before }\end{array}$ & $1.88 \pm 0.64$ & $1.75 \pm 0.71$ & .370 & 0.717 \\
ROSC & & & & \\
$\begin{array}{l}\text { Duration of } \\
\text { CPR } \\
\text { before }\end{array}$ & $274.9 \pm 77.2$ & $267.3 \pm 81.5$ & .192 & 0.850 \\
$\begin{array}{l}\text { ROSC (s) } \\
24 h\end{array}$ & 8 & & & \\
survival & & 8 & NA & NA \\
GRI & $1.38 \pm 0.52$ & $2.50 \pm 0.76$ & 3.473 & 0.004 \\
\hline All
\end{tabular}

All indexes were compared by the independent-samples $T$ test. The values are mean \pm s.d. or number (n). CPR: cardiopulmonary resuscitation; ROSC: spontaneous circulation recovery. GRI: grade of renal injury. TH: therapeutic hypothermia group, $\mathrm{NH}$ : normothermia group.

\section{Hemodynamic parameters and the lactate level}

The HR in the TH group was lower than that in the $\mathrm{NH}$ group at ROSC $6 \mathrm{~h}$ and $12 \mathrm{~h}$ $(\mathrm{P}<0.001)$. Compared with the $\mathrm{NH}$ group, the $\mathrm{CO}$ in the TH group was also lower at ROSC $12 \mathrm{~h}$ $(P=0.022)$, but the value ultimately exceeded that in the $\mathrm{NH}$ group $(\mathrm{P}<0.001)$ at ROSC $24 \mathrm{~h}$. The lactate level in the $\mathrm{TH}$ group was lower than that in the $\mathrm{NH}$ group $\left(\mathrm{P}_{6 \mathrm{~h}}<0.001, \mathrm{P}_{12 \mathrm{~h}}=0.001\right.$, $\left.P_{24 h}<0.001\right)$ after ROSC. Between the two other groups, the HR in the TH group were lower than those in the SHAM group at ROSC $6 \mathrm{~h}$ and $12 \mathrm{~h}$ $\left(P_{6 h}=0.018, P_{12 h}<0.001\right)$; and CO were also lower from ROSC $1 \mathrm{~h}$ to $12 \mathrm{~h}(\mathrm{P}<0.001)$. The lactate level was higher after ROSC $\left(P_{1 h}<0.001, P_{6 h}=0.018\right.$, $\left.P_{12 h}=0.005\right)$. No significant differences were found in the MAP among the three groups during the experimental protocol (Figure 2). 

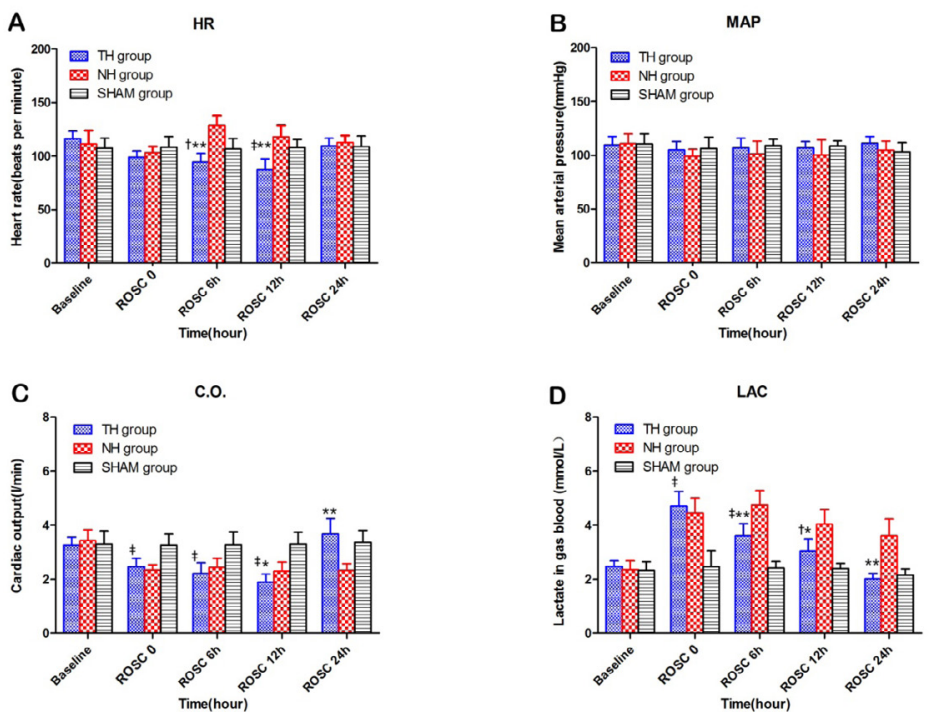

Figure 2 - Hemodynamic parameters and lactate in blood gas in three groups. HR (A); MAP (B): CO(C); Lac in blood gas (D). ROSC: restoration of spontaneous circulation; HR: heart rate; MAP: mean aortic pressure; C.O.: cardiac output; Lac: lactate. TH: therapeutic hypothermia group, NH: normothermia group; SHAM group: sham operation group. TH vs. $\mathrm{NH}:{ }^{*} \mathrm{P}<0.05,{ }^{*} \mathrm{P}<0.001$. TH vs. $\mathrm{SHAM:}+\mathrm{P}<0.05, \ddagger \mathrm{P}<0.001$.

Renal function and levels of NGAL and CysC

At baseline, the levels of $\mathrm{sCr}$, urine volume per hour and $\mathrm{Cc}$ were similar among all three groups. The $\mathrm{s} C r$ levels increased in $\mathrm{TH}$ and $\mathrm{NH}$ groups at ROSC $6 \mathrm{~h}$ compared with baseline $\left(P_{T H}=0.002, P_{N H}=0.001\right)$. Between the $\mathrm{TH}$ and $\mathrm{NH}$ groups, the $\mathrm{sCr}$ level was lower in the $\mathrm{TH}$ group $\left(P_{6 h}=0.027, P_{12 h}<0.001, P_{24 h}=0.023\right)$. The urine volume per hour in the $\mathrm{TH}$ group was higher than that in the $\mathrm{NH}$ group during cooling $\left(P_{6 h}<0.001, P_{12 h}<0.001\right)$. Also, the $\mathrm{Cc}$ in the $\mathrm{TH}$ group was obviously lower than that in the $\mathrm{NH}$ group after ROSC $\left(\mathrm{P}_{6 \mathrm{~h}}=0.001, \mathrm{P}_{12 \mathrm{~h}}<0.001\right.$, $\left.\mathrm{P}_{24 \mathrm{~h}}<0.001\right)$. Compared with the SHAM group, the $\mathrm{s} C r$ level and urine volume per hour in the TH group were significantly higher after ROSC, but the Cc was lower in this process (Figure 3).
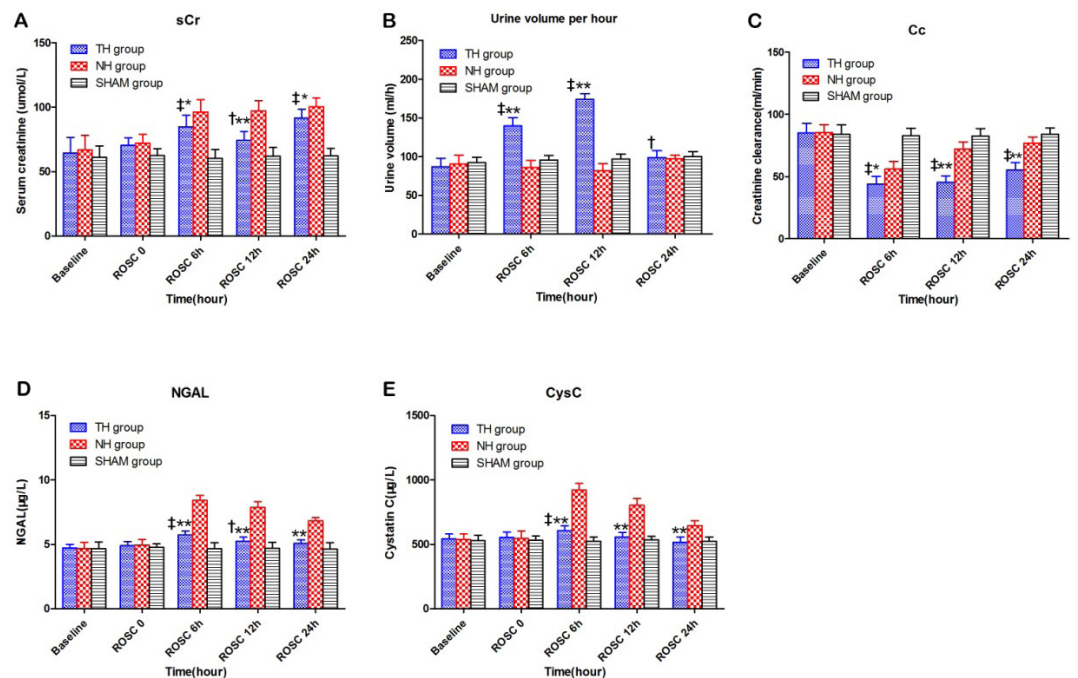

Figure 3 - Renal function and the levels of NGAL and CysC. ROSC: restoration of spontaneous circulation; NGAL: neutrophil gelatinase-associated lipocalin; TH: therapeutic hypothermia group, $\mathrm{NH}$ : normothermia group; SHAM group: sham operation group. TH vs. NH: ${ }^{*} P<0.05,{ }^{*} P<0.001$. TH vs. SHAM: $+P<0.05, \ddagger P<0.001$. 
For the biomarkers of kidney injury, there was no significant difference among all three groups at baseline and ROSC 0 . After ROSC, NGAL levels in the $\mathrm{NH}$ group were consistently higher than baseline $(\mathrm{P}<0.001)$. At ROSC $6 \mathrm{~h}$ and $12 \mathrm{~h}, \mathrm{NGAL}$ levels in the TH group were higher than baseline $\left(P_{6 h}=0.001, P_{12 h}=0.016\right)$, but the levels were consistently lower than those in the $\mathrm{NH}$ group after resuscitation $(\mathrm{P}<0.001)$. At ROSC 6h, Cys C levels in $\mathrm{TH}$ and $\mathrm{NH}$ groups were

Renal histopathology (Figure 4A-C) higher than baseline $\left(\mathrm{P}_{\mathrm{TH}}<0.001, \mathrm{P}_{\mathrm{NH}}<0.001\right)$. Also, CysC levels in the $\mathrm{NH}$ group continued to decrease throughout experimentation after ROSC, but were always higher than those in the TH group at ROSC 24h $(P<0.001)$. Compared with the SHAM group, NGAL levels in the TH group were higher at ROSC $6 \mathrm{~h}$ and $12 \mathrm{~h}\left(\mathrm{P}_{6 \mathrm{~h}}<0.001, \mathrm{P}_{12 \mathrm{~h}}=0.015\right)$, but $\mathrm{Cys} \mathrm{C}$ showed a higher level only at ROSC 6h $(P<0.001)$.

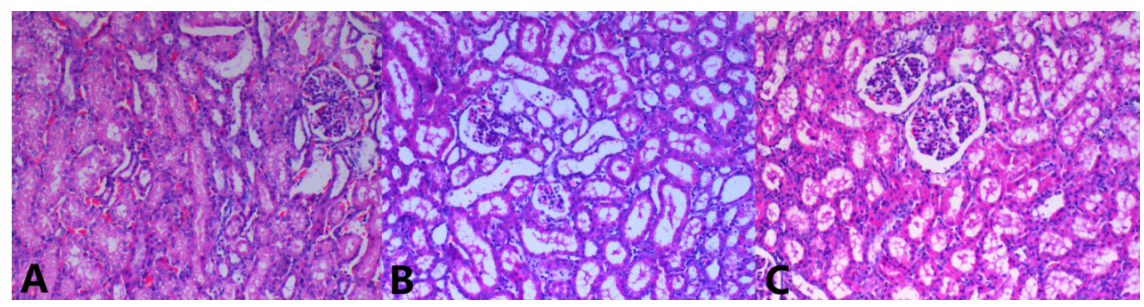

Figure 4 - The comparison of kidney from (A) TH group; (B) NH group; (C) SHAM group. Original magnification: $\times 200$. Interstitial edema, renal tubular expansion, epithelial cell and glomerular atrophy, and inflammatory cells infiltration were observed in the $\mathrm{NH}$ group. Compared to $\mathrm{NH}$ group, the $\mathrm{TH}$ group showed lighter tissue damage. The sham group showed normal structure. $\mathrm{TH}$ : therapeutic hypothermia group, $\mathrm{NH}$ : normothermia group; SHAM group: sham operation group.

Under light microscopy, the grade of renal injury was divided from 0 to 3 (Table 2). Compared with the $\mathrm{TH}$ group, renal injury was prominent in the $\mathrm{NH}$ group $(\mathrm{P}=0.004)$. Interstitial edema, expansion of renal tubules, atrophy of epithelial cells and glomeruli, and infiltration of inflammatory cells were observed. The sham group showed a normal structure whose GRI was $0.25 \pm 0.46$ and lower than that in the $\mathrm{TH}$ group $(P<0.001)$.

\section{- Discussion}

The present study showed that mild hypothermia had a protective effect on renal function after resuscitation compared with standard treatment. There were conflicting results among the indices of renal function, but the biomarkers of acute kidney injury and pathologic examination supported this finding. Impaired renal function could be confirmed through elevation of $\mathrm{sCr}$ levels in $\mathrm{TH}$ and NH groups after ROSC. This potential impact of ischemia/reperfusion injury on kidneys has been repeatedly confirmed in the past $^{2,16}$. In present study, compared with the $\mathrm{NH}$ group, mild hypothermia reduced the $\mathrm{sCr}$ level and increased urine output after ROSC, which may be considered to be signs of improving renal function. However, the reduced $\mathrm{Cc}$ under hypothermia suggested the opposite view. According to previous research, a possible explanation may be that the increase in renal vascular resistance during hypothermia reduced renal blood flow, and the spurious improvement in $\mathrm{sCr}$ level may be derived from a consecutive reduction in creatinine release from muscles ${ }^{7,17}$. In addition, considering the 
effects on hemodynamics by hypothermia, the decline in $\mathrm{CO}$ may reduce renal blood flow, but the lower lactate level in the TH group reflected the reduction of systemic oxygen consumption concurrently ${ }^{18,19}$. In fact, hypothermia usually causes changes in the systemic state, so neither of serum creatinine or urine output is sufficient to predict the true trend of renal function and assess the result of therapy under this condition ${ }^{7,15}$.

Relative to the above confusing results, using biomarkers to assess hypothermia treatment appears to be more clarity and reliable. Both NGAL and CysC are sensitive for the prediction of moderate-to-severe kidney injury in the early stage after ROSC ${ }^{20-22}$.NGAL is expressed in epithelial cells in different organs, and is considered to be an inflammatory mediator whose expression is upregulated in tubular injury. In CA patients, NGAL measured in blood within $4 \mathrm{~h}$ after ROSC is a predictor of $\mathrm{AKI}^{20}$. In present study, NGAL levels in the $\mathrm{NH}$ group were consistently higher than baseline after ROSC, and the TH group showed relatively lower levels and was close to the baseline value at ROSC 24h. These findings suggest that mild hypothermia may inhibit the inflammatory reaction during renal ischemia/reperfusion injury.

The CysC level usually increased within ROSC 2 to 3 hours in serum ${ }^{23}$, and is determined only by the glomerular filtration rate. CysC production in the body is a stable process that is not influenced by the constitution of the body ${ }^{23}$, such as inflammation or diet. In our study, CysC levels in the TH group showed only a transient increase after ROSC, which was different from the sustained rise observed in the $\mathrm{NH}$ group. It suggested that mild hypothermia can attenuate the impairment to glomerular function by reperfusion.

On the other hand, NGAL and CysC can be regarded as promising biomarkers, whose trends were in accordance with pathologic findings. In our results, levels of NGAL and CysC were lower even after rewarming in the $\mathrm{TH}$ group, which showed a corresponding lower GRI in a semi-quantitative pathological analysis. Infiltration of fewer inflammatory cells was observed in the TH group under light microscopy. Taken together, the protective effects of mild hypothermia in renal ischemia/ reperfusion injury were distinct. Although the exact mechanisms remain still unclear, it may be the result of the synthetic regulate, concerned with improving balance between tissue oxygen supply and demand, enhancing systemic anti-inflammatory reaction ${ }^{9}$ and lower levels of lactic acid ${ }^{24}$.

The present study had three main limitations. First, our experiments were conducted on healthy animals, but the changes occurring in pathophysiologic conditions may be different. Second, there was a lack of monitoring of renal blood flow that may be helpful to discuss the influence of mild hypothermia on the kidneys. Finally, a more objective assessment of renal function may necessitate a much longer observation period.

\section{Conclusion}

The mild hypothermia can protection against renal ischemia/reperfusion injury after resuscitation.

\section{References}

1. Adrie $\mathrm{C}$, Adib-Conquy $\mathrm{M}$, Laurent I, Monchi M, Vinsonneau C, Fitting C, Fraisse $F$, Dinh-Xuan AT, Carli P, Spaulding C, Dhainaut JF, Cavaillon JM. Successful cardiopulmonary resuscitation after cardiac arrest as a "sepsislike" syndrome. Circulation. 2002;106:5628. PMID: 12147537.

2. Domanovits $H$, Schillinger $M$, Müllner $M$, Thoennissen J, Sterz F, Zeiner A, Druml W. Acute renal failure after successful cardiopulmonary resuscitation. Intensive Care Med. 2001;27:1194-9. PMID: 11534568. 
3. Mattana J, Singhal PC. Prevalence and determinants of acute renal failure following cardiopulmonary resuscitation. Arch Intern Med. 1993;153:235-9. PMID: 8422211.

4. Moore EM, Nichol AD, Bernard SA, Bellomo R. Therapeutic hypothermia: benefits, mechanisms and potential clinical applications in neurological, cardiac and kidney injury. Injury. 2011 Sep;42(9):843-54. PMID: 21481385.

5. Bernard SA, Gray TW, Buist MD, Jones BM, Silvester W, Gutteridge G, Smith K. Treatment of comatose survivors of outof-hospital cardiac arrest with induced hypothermia. N Engl J Med. 2002;346:55763. PMID: 21481385.

6. The Hypothermia after Cardiac Arrest Study Group. Mild therapeutic hypothermia to improve the neurologic outcome after cardiac arrest. N Engl J Med. 2002;346:54956. PMID: 11856793.

7. Zeiner A, Sunder-Plassmann G, Sterz F, Holzer M, Losert $H$, Laggner AN, Müllner M. The effect of mild therapeutic hypothermia on renal function after cardiopulmonary resuscitation in men. Resuscitation. 2004;60:253-61. PMID: 15050756.

8. Delbridge MS, Shrestha BM, Raftery AT, El Nahas AM, Haylor JL. The effect of body temperature in a rat model of renal ischemia-reperfusion injury. Transplant Proc. 2007;39:2983-5. PMID: 18089305.

9. Polderman KH. Mechanisms of action, physiological effects, and complications of hypothermia. Crit Care Med. 2009;37:S186202. PMID: 19535947.

10.Peng ZY. The biomarkers for acute kidney injury: a clear road ahead?

J Transl Int Med. 2016 Sep 1;4(3):95-8. PMID: 28191528.

11.World Medical Association. World Medical Association Declaration of Helsinki: Ethical Principles for Medical Research Involving Human Subjects. JAMA. 2013;310(20):21914. PMID: 24141714.

12.Zhang $\mathrm{Q}$, Li C. Combination of epinephrine with esmolol attenuates post-resuscitation myocardial dysfunction in a porcine model of cardiac arrest. PLoS ONE. 2013;8(12):e82677. PMID: 24367539.

13.Wu J, Li C, Liu Z. A comparison of two types of chest compressions in a porcine model of cardiac arrest. Am J Emerg Med.
2009;27:823-9. PMID: 19683111.

14.Wang S, Li C, Ji X, Yang L, Su Z, Wu J. Effect of continuous compressions and 30:2 cardiopulmonary resuscitation on global ventilation/perfusion values during resuscitation in a porcine model. Crit Care Med. 2010;38:2024-30. PMID: 20683258.

15. Mei X, Hang CC, Wang S, Li CS, Yu ZX. Renal doppler and novel biomarkers to assess acute kidney injury in a swine model of ventricular fibrillation cardiac arrest. Chin Med J (Engl). 2015 Nov 20;128(22):3069-75. PMID: 26608988.

16.Sandroni C, Dell'anna AM, Tujjar O, Geri G, Cariou A, Taccone FS. Acute kidney injury after cardiac arrest: a systematic review and meta-analysis of clinical studies. Minerva Anestesiol. 2016 Sep;82(9):989-99. PMID: 26957119.

17.Broman $M$, Källskog $O$. The effects of hypothermia on renal function and haemodynamics in the rat. Acta Physiol Scand. 1995;153:179-84. PMID: 7778458.

18. Harvey RB. Effect of temperature on function of isolated dog kidney. Am J Physiol. 1959 Jul;197(1):181-6. PMID: 13661419.

19.Levy MN. Oxygen consumption and blood flow in the hypothermic, perfused kidney. Am J Physiol. 1959 Nov.;197:1111-4. PMID: 14416432.

20.Park SO, Ahn JY, Lee YH, Kim YJ, Min YH, Ahn HC, Sohn YD, Park SM, Oh YT, Shin DH. Plasma neutrophil gelatinase-associated lipocalin as an early predicting biomarker of acute kidney injury and clinical outcomes after recovery of spontaneous circulation in out-of-hospital cardiac arrest patients. Resuscitation. 2016;101:84-90. PMID: 26826562.

21.Mahboub P, Ottens $P$, Seelen $M$, 't Hart N, Van Goor H, Ploeg R, Martins PN, Leuvenink $\mathrm{H}$. Gradual rewarming with gradual increase in pressure during machine perfusion after cold static preservation reduces kidney ischemia reperfusion injury. PLoS One. 2015 Dec 2;10(12):e0143859. PMID: 26630031.

22.Su Song, Marko Meyer, Tobias R, Wilde B, Feldkamp T, Assert R, Wu K, Kribben A, Witzke O. Serum cystatin $C$ in mouse models: a reliable and precise marker for renal function and superior to serum creatinine. Nephrol Dial Transplant. 2009;24:1157-61. PMID: 19004848. 
23. Hang CC, Li CS, Wu CJ, Yang J. Acute kidney injury after cardiac arrest of ventricular fibrillation and asphyxiation swine model. Am J Emerg Med. 2014 Mar;32(3):208-15. PMID: 24361141.
24.Toraman F, Evrenkaya S, Yuce M, Aksoy N, Karabulut $\mathrm{H}$, Bozkulak Y, Alhan C. Lactic acidosis after cardiac surgery is associated with adverse outcome. Heart Surg Forum. 2004;7(2):E155-9. PMID: 15138095.

\section{Correspondence:}

Prof. Chun-Sheng Li

Department of Emergency Medicine Capital

Medical University

Beijing, China

Phone: +8610-85231051

2000ywei@sina.com

Received: Mar 14, 2017

Review: May 17, 2017

Accepted: June 19, 2017
Conflict of interest: none

Financial source: none
${ }^{1}$ Research performed at Beijing Key Laboratory of Cardiopulmonary Cerebral Resuscitation, China. 